\title{
Frequency of glutathione-S-transferase null-M1 and null-T1 genotypes among the Turabah population in Saudi Arabia
}

\author{
A.A. Mansour ${ }^{1,2}$, O.M. Saleh ${ }^{2,3}$, T. Askar ${ }^{2}$, A.M. Salim² and A. Merganiं ${ }^{2}$ \\ ${ }^{1}$ Department of Biology, Faculty of Agriculture, Ain Sham University, \\ Egypt and College of Applied Medical Sciences-Turabah, Taif University, Saudi Arabia \\ ${ }^{2}$ Department of Biology, College of Applied Medical Sciences-Turabah, \\ Taif University, Saudi Arabia \\ ${ }^{3}$ National Center for Radiation Research and Technology, Cairo, Egypt \\ Corresponding author: A. Mergani \\ E-mail: a.alahmar@tu.edu.sa / ad_merg@hotmail.com
}

Genet. Mol. Res. 14 (4): 16863-16871 (2015)

Received June 19, 2015

Accepted September 2, 2015

Published December 14, 2015

DOI http://dx.doi.org/10.4238/2015.December.14.13

ABSTRACT. Glutathione-S-transferases (GST) are key phase II detoxifying enzymes that play critical roles in protection against products of oxidative stress and against electrophiles. Glutathione S-transferase mu (GST-M1) and theta (GST-T1) are isoforms of glutathione transferase enzymes that participate in the metabolism of a wide range of chemicals. Deletion variants that are associated with a lack of enzyme function exist at both these loci. The frequencies of homozygous GSTM1 and GSTT1 deletion carriers are very high in most of the populations studied to date. The aim of this study was to investigate the frequencies of GSTM1 and GSTT1 genotypes among the Turabah population in Saudi Arabia in comparison with the data published for some other Arabic populations. The subjects consisted of 164 unrelated healthy individuals from the Turabah population. GST genotyping was performed by multiplex polymerase chain reaction-based 
methods. The GSTM1 deletion homozygosity was $56.1 \%$ and GSTT1 deletion homozygosity was $20.7 \%$, while the GSTM1 and GSTT1 doubledeletion homozygosity was $11.0 \%$. Comparison with published data from Bahraini, Lebanese, and Tunisian populations demonstrated no significant difference for GSTM1 between these populations. The GSTT1 null-allele frequency was significantly lower than those for the Lebanese and Tunisian populations $(P=0.001)$ but similar to that for the Bahraini population ( $P$ $=0.099$ ). Characterization of GST genetic polymorphisms in the Saudi population may aid in genetic studies on the association of GSTM1 and GSTT1 polymorphisms with disease risks and the pharmacogenetics of chemotherapy.

Key words: Glutathione S-transferase; Genetic polymorphisms; Turabah; SA

\section{INTRODUCTION}

The human body is exposed to a wide range of xenobiotics, ranging from food components or environmental toxins to synthetic and natural xenoestrogens, in addition to exposure to its own steroidal estrogens. These synthetic chemicals include a number of chlorinated organic compounds, such as the insecticides kepone, dieldrin, and DDT and its metabolites and some polychlorinated biphenyl (PCB) congeners. More recently, nonchlorinated compounds used as antioxidants and plasticizers were found to be estrogenic and carcinogenic (Soto et al., 1997; Farmer et al., 2003).

The human body has developed complex enzymatic mechanisms to detoxify these substances. These mechanisms exhibit significant individual variability and are affected by environmental, lifestyle, and genetic factors (Liska, 1998). The detoxification process involves two phases: functionalization, which uses oxygen to form a reactive site, and conjugation, which results in addition of a water-soluble group to the reactive site to be excreted in urine. These two steps are termed as phase I and phase II detoxification, respectively (Estabrook, 1996). Several genes involved in phase I and phase II have recently been cloned and identified in humans. Many of them show polymorphisms that may cause alteration in expression, function, and activity and have been suggested to contribute to individual cancer susceptibility as genetic modifiers of cancer risk (Adam et al. 2003; Chacko et al., 2005; Habuchi, 2006).

Glutathione S-transferases (GSTs) are phase II xenobiotic metabolizing enzymes that are directly involved in catalyzing the conjugation of reactive intermediates of electrophilic xenobiotics with glutathione. GSTs are composed of four major groups: GSTA $(\alpha), \operatorname{GSTT1}(\theta), \operatorname{GSTM} 1(\mu)$, and GSTP1 $(\pi)$ (Lai and Shields, 1999; Geisler and Olshan, 2001). The enzyme encoded by the GSTT1 gene may also be active in endogenous mutagenic processes. GST-M1 and GST-T1 are isoforms of GSTs that participate in the metabolism of a wide range of chemicals, including possible carcinogens (Hayes and Pulford, 1995; Shukla et al., 2012; Matic et al., 2013; Acevedo et al., 2014). The known substrates for the GST-M1 enzyme include reactive peroxide intermediates generated from the activation of polycyclic aromatic hydrocarbons by cytochrome P450 enzymes. Deletion variants that are associated with a lack of enzyme function exist at both these loci. Individuals who are carriers of homozygous deletions in the GSTM1 of GSTT1 genes may have impaired ability to metabolically eliminate carcinogenic compounds and may therefore be at increased risk for 
cancer. GST enzymes play with glutathione peroxidases (GPx) an important role in the catabolism of glutathione (GSH), which is a potent antioxidant (contains a thiol group); this prevents damage to important cellular components caused by reactive oxygen species such as free radicals and peroxides (Danyelle, 2003).

The frequencies of homozygous GSTM1 and GSTT1 deletion carriers is very high (i.e., 20-50\%) in most populations studied to date.

Epidemiological studies suggest that individuals who are homozygous null at the GSTM1 and GSTT1 loci (i.e., lack both copies of the GSTM1 and/or GSTT1 variants of the GST gene family) may have an increased risk of developing various types of neoplastic diseases, including cancers of the bladder, prostate, colon, skin, lung, and stomach (Strange and Fryer 1999; Altayli et al., 2009; Nock et al., 2009; Simic et al., 2009; Zhao et al., 2014). Therefore, knowledge regarding the inheritance of these susceptibility genes in different ethnic groups is very important for effective prevention of disease, especially cancer (Klusek and Gluszek, 2014).

This study focused on screening for double null mutations in GSTs mu and theta and determining their frequencies in the human population of Turabah in the western region of Saudi Arabia.

\section{MATERIAL AND METHODS}

\section{Study subjects}

Blood samples were collected from 164 unrelated individuals, who were born and were living in Turabah province, after obtaining their consent to participate in the study. The mean age of the study subjects was $44.87 \pm 10.46$ years; the minimum age was 30 years and the maximum was 60 years. From the study population, 98 (60\%) were men and $66(40 \%)$ were women. The study was approved by the Ministry of Health and Research Committee, Taif University.

\section{Genomic DNA extraction}

Genomic DNA was extracted from peripheral blood using standard procedures (Sambrook and Russel, 2001). Briefly, $5 \mathrm{~mL}$ blood was mixed with an equal volume of RBC lysis buffer (0.32 $\mathrm{M}$ sucrose, $5 \mathrm{mM} \mathrm{MgCl}, 10 \%$ Triton 100X, and $12 \mathrm{mM}$ Tris- $\mathrm{HCl}, \mathrm{pH} 8.0$ ) and allowed to sit for 5 min. The cell lysate was then centrifuged at $3000 \mathrm{rpm}$ and the upper layer was discarded. The pellet was resuspended in phosphate-buffered saline (PBS; $\mathrm{pH} 7.4$ ) and 1\% sodium dodecyl sulfate, and then digested with $0.2 \mathrm{mg} / \mathrm{mL}$ proteinase $\mathrm{K}$ at $55^{\circ} \mathrm{C}$ overnight. After digestion, the DNA was purified using phenol-chloroform extraction, followed by ethanol precipitation with 0.3 $\mathrm{M}$ potassium acetate, $\mathrm{pH}$ 5.2. The DNA was finally been stored in TE (10 mM Tris- $\mathrm{HCl}$ and $1 \mathrm{mM}$ EDTA, pH 8.0) until use.

\section{Genotyping of GSTM1 and GSTT1 polymorphisms}

A multiplex polymerase chain reaction (PCR)-based method and three sets of primers were used for identification of the GSTM1 and T1 genotypes (Chen, 1996).

The sequences for the GSTM1 forward and reverse primers were as follows: 
5'-gaactccctgaaaagctaaagcc-3' and 5'-gttgggctcaaatatacggtgg-3', respectively (Bell et al., 1993). The sequences for the GSTT1 forward and reverse primers were 5'-ttccttactggtcctcacatctc-3' and 5'-tcaccggatcatggccagca-3', respectively (Pemble et al., 1994). To ensure that a null genotype was due to the absence of GSTM1 and GSTT1 alleles rather than failure of PCR, we coamplified human $\beta$-globin as the internal control using the primers 5 '-caacttcatccacgttcacc- 3 ' and 5'-gaagagccaaggacaggtac-3'. The PCR was carried out in a Applied Biosystem 9700 Thermocycler in a 25- $\mu \mathrm{L}$ reaction mixture containing 50-100 ng genomic DNA; $1.6 \mathrm{mM}$ dNTPs; $200 \mathrm{nM}$ each GSTM1, GSTT1, and $\beta$-globin primer; $10 \mathrm{mM}$ Tris/HCl, pH 9.2; $50 \mathrm{mM} \mathrm{KCl} ; 2 \mathrm{mM}$ $\mathrm{MgCl}_{2}$; and $1.5 \mathrm{U}$ Taq polymerase. Cycling conditions were as follows: an initial $4 \mathrm{~min}$ at $93^{\circ} \mathrm{C}$ for loading and denaturation, followed by $45 \mathrm{~s}$ at $61^{\circ} \mathrm{C}$ and $90 \mathrm{~s}$ at $72^{\circ} \mathrm{C}$. This was followed by an additional 36 cycles of $93^{\circ} \mathrm{C}$ for $30 \mathrm{~s}, 63^{\circ} \mathrm{C}$ for $45 \mathrm{~s}$, and $72^{\circ} \mathrm{C}$ for $90 \mathrm{~s}$. Negative controls were includes for all amplifications; the reaction mixtures for these contained all the components except the DNA template. The PCR products were resolved on an ethidium bromide-stained 1.7\% 1:1 NuSieve/MP agarose gel together with a DNA molecular weight marker. Amplification with $\beta$-globin primers produced a 268 -bp band indicating that the PCR is reliable.

The presence of a 215-bp band indicated that this subject is primers for GSTM1 homozygote or heterozygote, while its absence in the presence of the internal control band indicated the null GSTM1. The presence of a 480-bp band indicated that the subject was homozygote or heterozygote for GSTT1, while its absence in the presence of the internal control band indicated the null GSTT1.

\section{Statistical analysis}

The allele/genotype frequencies of the two polymorphisms were tested for Hardy-Weinberg equilibrium using the $\chi^{2}$ test. The same test was used to compare the gene frequencies among the study population and published data from three Arabic populations, namely, Bahrainis, Jordanians, and Tunisians. The differences between the study population and each of these populations were considered significant if the $\mathrm{P}$ value did not exceed 0.05 .

\section{RESULTS}

\section{Frequencies of genotypes of the GSTM1 and GSTT1 polymorphisms among the study population}

The frequencies of the genotypes of the GSTM1 and GSTT1 polymorphisms were screened using multiplex PCR in 167 individuals. This method only differentiated between homozygous deletion (null-genotype) of both polymorphisms and wild-type and heterozygous genotypes (positive genotype), as shown in Figure 1. The frequencies of GSTM1 and GSTT1 null-genotypes in the study population, as shown in Table 1, were $92(56.1 \%)$ and $34(20.7 \%)$, respectively. Genotyping showed that $74(45.1 \%)$ individuals had the GSTM1 null-genotype and were GSTT1 positive while only $16(9.7 \%)$ showed the GSTM1 positive and GSTT1 nullgenotype. The double deletion was found in 18 individuals (11\%). The GSTM1 and GSTT1 nullallele frequencies were 0.749 and 0.448 , respectively. 


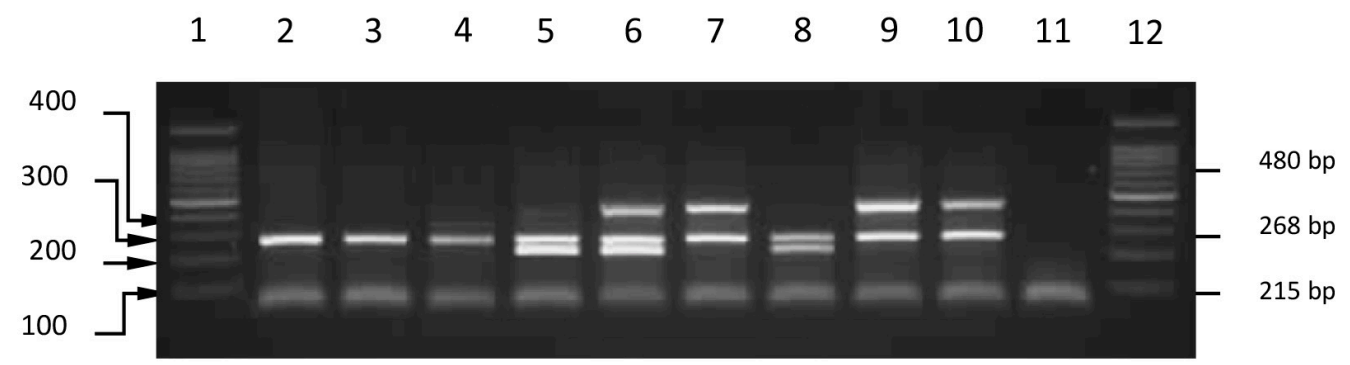

Figure 1. Genotyping of GSTM1 and GSTT1 polymorphisms by a multiplex PCR-based method. Lanes 1 and 12: 100-bp DNA marker. Lane 11: negative control. Lanes 2, 3 and 4: individuals with null-GSTM1/null-GSTT1 genotypes. Lanes 5 and 8: individuals with positive GSTM1/null-GSTT1 genotypes. Lane 6: individual with positive GSTM1/ positive GSTT1 genotypes. Lanes 7, 9 and 10: individuals with null-GSTM1/positive GSTT1 genotypes.

Table 1. Frequencies of GSTM1 and GSTT1 polymorphisms in the study population.

\begin{tabular}{|c|c|c|c|c|}
\hline \multirow[t]{2}{*}{ GST } & & \multicolumn{2}{|c|}{ M1 } & \multirow[t]{2}{*}{ Total (\%) } \\
\hline & & Null (\%) & Positive (\%) & \\
\hline $\mathrm{T1}$ & $\begin{array}{l}\text { Null (\%) } \\
\text { Positive (\%) }\end{array}$ & $\begin{array}{l}18(11) \\
74(45.1) \\
92(56.1)\end{array}$ & $\begin{array}{l}16(9.7) \\
56(34.2) \\
72(43.9)\end{array}$ & $\begin{array}{r}34(20.7) \\
130(79.3) \\
164(100)\end{array}$ \\
\hline
\end{tabular}

\section{Frequencies of the GSTM1 and GSTT1 polymorphisms in the study population in comparison to those in the three Arabic populations}

Figure 2 shows the frequencies of the null genotypes and null alleles of both polymorphisms among the study population in the present study and those of the Bahraini, Lebanese, and Tunisian populations that were published by Salem et al. (2011). Both GSTT1 null- and double-deletion frequencies in the study population were lower than those in the other three populations. Comparison of the gene frequencies of the study population with those of the Bahraini, Lebanese, and Tunisian populations demonstrated that there was no significant difference in the gene frequencies between the study and Bahraini populations for GSTM1 $(P=0.059)$ and GSTT1 ( $P=0.146$; Table 2). Comparison of GSTM1 and GSTT1 gene frequencies in the study population with those in the Lebanese and Tunisian populations indicated that were significant differences in GSTT1 genotypes between the study population and both these populations $(P=0.0001$ and 0.0001 , respectively) but not for the GSTM1 genotypes $(P=0.303$ and 0.327 , respectively; Tables 3 and 4$)$.

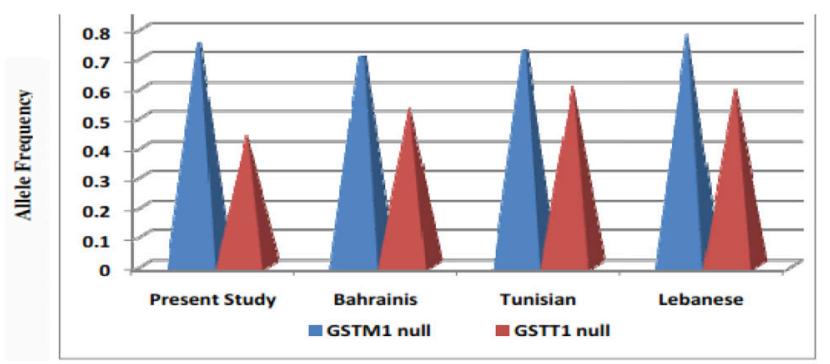

Figure 2. GSTM1 and GSTT1-null allele frequencies in the study population. 
Table 2. Comparison of GSTM1 and GSTT1 genotype frequencies between the study and Bahraini populations.

\begin{tabular}{|c|c|c|c|c|c|}
\hline \multirow[t]{3}{*}{ Population } & \multirow[t]{3}{*}{$\mathrm{N}$} & \multicolumn{4}{|c|}{ Genotype frequency (\%) } \\
\hline & & \multicolumn{2}{|c|}{ GSTM1 } & \multicolumn{2}{|c|}{ GSTT1 } \\
\hline & & Positive & Null & Positive & Null \\
\hline Present Study & 164 & $72(43.9)$ & $92(56.1)$ & $130(79.3)$ & $34(20.7)$ \\
\hline Bahrainis & 167 & $84(50.3)$ & $83(49.7)$ & 119 (71.3) & $48(28.7)$ \\
\hline$P$ value & & 0.146 & & 0.059 & \\
\hline
\end{tabular}

Table 3. Comparison of GSTM1 and GSTT1 genotype frequencies between the study and Lebanese populations.

\begin{tabular}{lccccc}
\hline Population & $\mathrm{N}$ & \multicolumn{3}{c}{ Genotype frequency (\%) } \\
\cline { 3 - 5 } & & \multicolumn{2}{c}{ GSTM1 } & Null & Positive \\
\cline { 3 - 6 } & & Positive & $92(56.1)$ & $130(79.3)$ & Null \\
\hline Present Study & 164 & $72(43.9)$ & $74(52.5)$ & $88(62.4)$ & $34(20.7)$ \\
Lebanese & 141 & $67(47.5)$ & & 0.001 & $53(37.6)$ \\
P value & & 0.303 & & \\
\hline
\end{tabular}

Table 4. Comparison of GSTM1 and GSTT1 genotype frequencies between the study and Tunisian populations.

\begin{tabular}{lccccc}
\hline Population & $N$ & \multicolumn{3}{c}{ Genotype frequency (\%) } \\
\cline { 3 - 5 } & & \multicolumn{2}{c}{ GSTM1 } & Null & Positive \\
\cline { 3 - 6 } & & Positive & $92(56.1)$ & $130(79.3)$ & Null \\
\hline Present Study & 164 & $72(43.9)$ & $118(52.5)$ & $117(62.9)$ & $34(20.7)$ \\
Tunisians & 186 & $68(36.6)$ & & 0.001 & $69(37.1)$ \\
P value & & 0.327 & & & \\
\hline
\end{tabular}

\section{DISCUSSION}

The human body is exposed to a wide range of xenobiotics, ranging from food components or environmental toxins to synthetic and natural xenoestrogens. The GST gene family consists of several genes that are located on different chromosomes. The expression of the GST genes has been reported to vary in different ethnic populations due to GST gene deletion polymorphisms. GSTM1 and GSTT1 deletion polymorphisms were found to be associated with relatively higher susceptibility to different malignancies and diseases in people sharing the same environment (Naveen et al., 2004; Garte et al., 2007).

In the present study, the frequency of the GSTM1 null genotype in the Saudi population was $56.1 \%$, which was similar to those reported in many other Arabic populations, that is, Bahrainis, Tunisian, Lebanese (Salem et al., 2011), and Egyptian (Hamdy et al., 2003) populations. The frequency of the GSTM1 null genotype in the present study was significantly higher than those in Asian populations, that is, Indian and Japanese (Kihara and Noda, 1999; Sharma et al., 2012) populations, and African populations, that is, Zimbabwean and Cameroonian populations (Piacentini et al., 2011). Likewise, the frequency of the GSTT1 nullgenotype in the Saudi population is $20.1 \%$. Comparison of the frequencies with those reported in other Arabic populations, that is, Bahraini, Tunisian, and Lebanese populations (Salem 
et al., 2011), demonstrated that the frequency was similar to that reported in Bahrainis but was significantly lower than those for the Tunisians and Lebanese $(P=0.0001$ and 0.0001 , respectively). Although both Tunisian and Lebanese populations are of Arabic ethnicity, these differences in GSTT1 null frequencies could be attributed to many factors. One reason could be the different environments inhabited by each population and the natural selection process that may affect the frequencies of genes that interact with environmental factors and common diseases in different population. Second, the Saudi population still preserved several Arabic social habits and behaviors. Almost all the Saudi subpopulations and tribes are living as small isolated and closed populations with firm restrictions on random genetic mating with high levels of consanguinity and inbreeding. Thus, mating between individuals in such subpopulations that are closely related (based on shared genes) is nearly the same as that between brothers and sisters, resulting in equal fertility for all genotype groups without selection and gene flow (or migration of individuals) in or out. This may result in increased homozygosity in these populations and may explain the high GSTM1 frequency observed, positive assortative mating and lower GSTT1 null genotypes and alleles, and negative assortative mating noted in the study population. The Saudi population may be similar to the Bahraini population but clearly differed from the Tunisian and Lebanese populations, which explains the significant differences between the study population and Tunisian and Lebanese populations. The positive assortative mating for the GSTM1 null genotype in this population may have a protective effect against common diseases in the study population or recently selected with changes in living standards, nutritional habits, and behavioral style during the last few decades, especially after the discovery and mining of petroleum in this area (Abu-Amero et al., 2006; Siraj et al., 2008; Saeed et al., 2013).

In conclusion, the observed frequencies of GSTM1 and GSTT1 null polymorphisms in the Saudi population in Turabah Province, Western region of Saudi Arabia, differed from those found in some other populations. Moreover, changes in living standards and behavioral style and habits are associated with increase in the frequencies of malignancies and metabolic diseases, and the susceptibility to disease incidences due to environmental pollutants will vary. Therefore, further and more detailed studies are highly recommended in this region and other regions in Saudi Arabia in order to identify the genetic as well as environmental factors related to predisposition to malignant and metabolic diseases. Thus, accumulation of such data will provide a basic database for future clinical and preventive measures against these diseases.

\section{Conflicts of interest}

The authors declare no conflict of interest.

\section{ACKNOWLEDGMENTS}

We would like to thank all the participants for consenting to participate in this study and the clinicians and laboratory staff at Turabah General Hospital for their support in facilitating the selection of study participants. This article is a part of a research project to study the Genetic of Metabolic Diseases that was funded by Research Center, Taif University, Saudi Arabia. 


\section{REFERENCES}

Abu-Amero KK, Al-Boudari OM, Mohamed GH and Dzimiri N (2006). T null and M null genotypes of the glutathione S-transferase gene are risk factor for CAD independent of smoking. BMC Med. Genet. 7: 38.

Acevedo CA, Quiñones LA, Catalán J, Cáceres DD, et al. (2014). Impact of CYP1A1, GSTM1, and GSTT1 polymorphisms in overall and specific prostate cancer survival. Urol. Oncol. 32: 280-290.

Adam PJ, Berry J, Loader JA, Tyson KL, et al. (2003). Arylamine N-acetyltransferase-1 is highly expressed in breast cancers and conveys enhanced growth and resistance to etoposide in vitro. Mol. Cancer Res. 1: 826-835.

Altayli E, Gunes S, Yilmaz AF, Goktas S, et al. (2009). CYP1A2, CYP2D6, GSTM1, GSTP1, and GSTT1 gene polymorphisms in patients with bladder cancer in a Turkish population. Int. Urol. Nephrol. 41: 259-266.

Bell DA, Taylor JA, Paulson DF, Robertson CN, et al. (1993). Genetic risk and carcinogen exposure: a common inherited defect of the carcinogen-metabolism gene glutathione S transferase M1 (GSTM1) that increases susceptibility to bladder cancer. JNCl 85: 1159-1164.

Chacko P, Joseph T, Mathew BS, Rajan B, et al. (2005). Role of xenobiotic metabolizing gene polymorphisms in breast cancer susceptibility and treatment outcome. Mutat. Res. 581: 153-163.

Chen CL, Liu Q and Relling MV (1996). Simultaneous characterization of glutathione S-transferase M1 and T1 polymorphisms by polymerase chain reaction in American whites and blacks. Pharmacogenetics 6: 187-191.

Estabrook RW (1996). Cytochromes P450: Metabolic and toxicological aspects. CRC Press, Inc, Boca Raton, 3-28.

Farmer PB, Singh R, Kaur B, Sram RJ, et al. (2003). Molecular epidemiology studies of carcinogenic environmental pollutants. Effects of polycyclic aromatic hydrocarbons (PAHs) in environmental pollution on exogenous and oxidative DNA damage. Mutat. Res. 544: 397-402.

Garte S, Taioli E, Popov T, Kalina I, et al. (2007). Role of GSTT1 deletion in DNA oxidative damage by exposure to polycyclic aromatic hydrocarbons in humans. Int. J. Cancer 120: 2499-2503.

Geisler SA and Olshan AF (2001). GSTM1, GSTT1, and the risk of squamous cell carcinoma of the head and neck: a miniHuGE review. Am. J. Epidemiol. 154: 95-105.

Habuchi T. (2006). Common genetic polymorphisms and prognosis of sporadic cancers: prostate cancer as a model. Future Oncol 2: 233-245.

Hamdy SI, Hiratsuka M, Narahara K, Endo N, et al. (2003). Genotype and allele frequencies of TPMT, NAT2, GST, SULT1A1 and MDR-1 in the Egyptian population. Br. J. Clin. Pharmacol. 55: 560-569.

Hayes JD and Pulford DJ (1995). The glutathione S-transferase supergene family: regulation of GST and contribution of the isoenzymes to cancer chemoprotection and drug resistance. Crit. Rev. Biochem. Mol. Biol. 30: 445-600.

Kihara M and Noda K (1999). Lung cancer risk of the GSTM1 null genotype is enhanced in the presence of the GSTP1 mutated genotype in male Japanese smokers. Cancer. Lett. 137: 53-60.

Klusek J and Gluszek S (2014). GST gene polymorphisms and the risk of colorectal cancer development. Contemp. Oncol. (Pozn) 18: 219-221.

Lai $C$ and Shields PG. (1999). The role of interindividual variation in human carcinogenesis. J. Nutr. 129: 552S-555S.

Liska DJ (1998). The detoxification enzyme systems. Altern. Med. Rev. 3: 187-198.

Matic M, Pekmezovic T, Djukic T, Mimic-Oka J, et al. (2013). GSTA1, GSTM1, GSTP1, and GSTT1 polymorphisms and susceptibility to smoking-related bladder cancer: a case-control study. Urol. Oncol. 31: 1184-1192.

Naveen AT, Adithan C, Padmaja N, Shashindran CH, et al. (2004). Glutathione S-transferase M1 and T1 null genotype distribution in South Indians. Eur. J. Clin. Pharmacol. 60: 403-406.

Nock NL, Bock C, Neslund-Dudas C and Beebe-Dimmer J (2009). Polymorphisms in glutathione S-transferase genes increase risk of prostate cancer biochemical recurrence differentially by ethnicity and disease severity. Cancer Causes Control. 20: 1915-1926.

Pemble S, Schroeder KR, Spencer SR, Meyer DJ, et al. (1994). Human glutathione S-transferase theta (GSTT1): cDNA cloning and the characterization of a genetic polymorphism. Biochem. J. 300: 271-6.

Piacentini S, Polimanti R, Porreca F, Martínez-Labarga C, et al. (2011). GSTT1 and GSTM1 gene polymorphisms in European and African populations. Mol. Biol. Rep. 38: 1225-1230.

Saeed HM, Alanazi MS, Nounou HA, Salaby MA, et al. (2013). Cytochrome P450 1A1, $2 E 1$ and GSTM1 gene polymorphisms and susceptibility to colorectal cancer in the Saudi population. Asian Pac. J. Cancer Prev. 14: 3761-3768.

Salem AH, Yaqoob A, Ali M, Handu S, et al. (2011). Genetic polymorphism of the glutathione S-transferase M1 and T1 genes in three distinct Arab populations. Dis. Markers 31: 311-316.

Sambrook J, Russell DW (2001). Molecular cloning: a laboratory manual. Cold Spring Harbor Press, Cold Spring Harbor, NY.

Sharma A, Pandey A, Sardana S, Sehgal A, et al. (2012). Genetic polymorphisms of GSTM1 and GSTT1 genes in Delhi and comparison with other Indian and global populations. Asian Pac. J. Cancer Prev. 13: 5647-5652.

Shukla D, Dinesh Kale A, Hallikerimath S, Vivekanandhan S, et al. (2012). Genetic polymorphism of drug metabolizing enzymes (GSTM1 and CYP1A1) as risk factors for oral premalignant lesions and oral cancer. Biomed. Pap. Med. Fac. 
Univ. Palacky. Olomouc. Czech Repub. 156: 253-259.

Simic T, Savic-Radojevic A, Pljesa-Ercegovac M, Matic M, et al. (2009). Glutathione S-transferases in kidney and urinary bladder tumors. Nat. Rev. Urol. 6: 281-289.

Siraj AK, Ibrahim M, Al-Rasheed M, Abubaker J, et al. (2008). Polymorphisms of selected xenobiotic genes contribute to the development of papillary thyroid cancer susceptibility in Middle Eastern population. BMC Med. Genet. 9: 61.

Soto AM1, Fernandez MF, Luizzi MF, Oles Karasko AS, et al. (1997). Developing a marker of exposure to xenoestrogen mixtures in human serum. Environ. Health Perspect. 105 (Suppl 3): 647-654.

Strange RC and Fryer AA (1999). The glutathione S-transferases: influence of polymorphism on cancer susceptibility. IARC Sci. Publ. 148: 231-249.

Townsend DM, Tew KD and Tapiero H (2003). The importance of glutathione in human disease. Biomed. Pharmacother 57: 145-155.

Zhao Y, Zeng J, Zhang Y, Lu S, et al. (2014). GSTM1 polymorphism and lung cancer risk among East Asian populations: a meta-analysis. Tumour Biol. 35: 6493-6500. 